\title{
Is This the End for EVAR?
}

\author{
Raman Uberoi ${ }^{1}\left[\right.$ Michael Jenkins ${ }^{2}$
}

Received: 10 October 2019/Accepted: 17 October 2019/Published online: 14 November 2019

(C) Springer Science+Business Media, LLC, part of Springer Nature and the Cardiovascular and Interventional Radiological Society of Europe (CIRSE) 2019

The endovascular aneurysm repair (EVAR) trials showed conclusively the significant initial benefit of EVAR compared to standard open surgical repair, in particular better 30 day mortality of 1.6 vs. $4.7 \%$ and lower major cardiac, respiratory, haemorrhagic and renal complications. These results were replicated by several other studies in different healthcare systems including the OVER, DREAM and ACE trials. This of course was not surprising given the less invasive nature of the new procedure [1].

Although the costs of EVAR were shown to be higher, with some additional unique complications such as endoleaks, EVAR became established as a viable alternative for the treatment of patients with unruptured abdominal aortic aneurysms (AAA) and was recognised by national institute for health and care excellence (NICE) in the UK. Industry partners listened to clinician's needs and devices became easier to use and more accurate to deploy. With increasing confidence in EVAR, many operators started placing stentgrafts in virtually all patients including those outside the instructions for use (IFU) with increasingly difficult anatomy, in some centres achieving a $>90 \%$ EVAR rate. Industry responded to these demands in an effort to make devices applicable to an ever wider subset of patients and with that has come a reduction in profile and evolution of the original design concept. Newer alloys such as Nitinol, repositionable devices, shorter $\mathrm{Z}$ stents, ring or spiral type stents and wider fabric gaps have been developed to increase conformability and flexibility. This focus was initially directed at wider applicability and backed by good early outcomes, but durability was not considered with few studies reporting outcomes beyond 3 years.

Amid the enthusiasm from both clinicians and patients, there were some early signs of possible trouble ahead. The Eurostar registry reported poorer outcomes and increased re-interventions in patients with large sacs and larger diameter proximal necks. Others went further with Jack Collin and John Murie entitling a 2002 editorial in the British Journal of Surgery, "Endovascular treatment of abdominal aortic aneurysm: A failed experiment". This early wakeup call highlighted the failure of EVAR to significantly reduce the overall rupture risk in the population treated, the failure rate of devices and the higher costs. However, at the time, there was no hard evidence to back this warning shot across EVAR's bows and the general enthusiasm for the procedure won through.

However, the long term follow-up data from some of the original EVAR trials have started to show some disturbing results [2-4]. The 3\% early EVAR survival benefit is lost by 4 years, and between 8 and 14 years there is a survival benefit to open surgery. This is as a result of an increase in both aneurysm related and overall mortality in the EVAR group. Late aortic ruptures account for the former and concerns regarding an increase in abdominal malignancies due at least in part to increased radiation exposure caused by numerous follow-up CT-examinations may have contributed to the latter. In

Raman Uberoi

raman.uberoi@ouh.nhs.uk

John Radcliffe Hospital, Oxford University Hospitals, Oxford, UK

2 Imperial College Healthcare NHS Trust, St Mary's Hospital, London, UK 
addition, costs have been shown to be significantly higher due in part to the need for continued follow-up and higher reintervention rates of around $16 \%$. When NICE did their costings using data from the ACE, DREAM and EVAR trials, the incremental cost-effectiveness ratio (ICER) was between $£ 48,990$ and 2.8 million per quality of life gain (QALY). The OVER trial was the only study assessed in isolation to show the potential to meet the $£ 20,000$ per QALY threshold with a high probability of $91 \%$ [5]. NICE's calculations, however, using all the available trials data, showed a $<1 \%$ probability of achieving this.

Proponents of EVAR argue that the trial data are historical and that both contemporary practice and today's devices have improved sufficiently to make the outcomes mentioned above obsolete. The appreciation of the need for a more proximal seal zone, a reduction in intervention for all type II endoleaks and moving to duplex surveillance may have an impact, but to date there is no high level evidence to support such claims. This has led to the recent controversial draft NICE guidelines on AAA management, where EVAR is no longer recommended for unruptured aneurysms and only in the context of research studies for juxta-renal or supra-renal aneurysms. NICE guidelines are highly valued and respected throughout the world so although this so far has only been released for consultation, it has resulted in a huge impact and major negative response from the world medical community.

Even if published in an unabridged form, most feel that implementation would be difficult. EVAR is a mature treatment within all vascular units in the local aortic networks (LANs) in the united kingdom (UK). Patients have embraced the minimally invasive advantages with faster recovery times. Despite there being patient representation on the NICE board, there is a feeling that the recommendations in their current form do not take account of patient choice and the greater importance an elderly population attaches to early benefit compared with what might happen in a decade's time. In a world where global information is available instantly, patients will no doubt feel they are entitled to at least consider treatment modalities, not only available, but often recommended as first choice in most European countries, Australia and the United states of America (USA). Counterintuitively, the draft NICE guidelines recommend EVAR as an option for emergency treatment in rupture patients (based on the IMPROVE results) which clearly would be difficult if operators no longer have experience of elective cases and potentially the full range of equipment necessary for all situations. The impact on hospital capacity also has to be considered. Many struggle with both hospital bed and critical care capacity, and the conversion of a proportion of AAA patients from EVAR to open surgery is likely to impact significantly on length of stay and critical care bed usage. Moreover, the UK AAA national screening programme (NAAASP) may also be at risk. Already, up to $20 \%$ of screen-detected AAA patients do not undergo intervention, mostly as a result of fitness. If this was increased further (inevitable if open surgery is the only treatment option), the utility, not to mention the cost-effectiveness of the NAAASP would have to be reconsidered.

Many of us of course still believe that EVAR has a major role still to play in the treatment of abdominal aortic aneurysms, but now appreciate that this cannot apply to all patients. However, as clinicians, we have failed to identify the cohort that will benefit long term. It is extremely unlikely that we will be able to set up another randomised trial for EVAR and even if we could, it would take a decade or more to produce meaningful results to satisfy durability concerns. Instead, we need to urgently review what we have in terms of published data to show which groups we believe will benefit from EVAR. The UK National Vascular Registry (NVR) was designed to report early outcomes and more recently provide information to support the NHS directed need for individual surgeon outcome data in the public domain. Most would now agree that 30 day mortality figures of 1-2\% make such outcome measures less relevant and we need to look at longitudinal data over the duration of a patient's life to gain a better insight into durability. Although possible, this is far more resource heavy and expensive, but clearly now necessary.

Whether we accept the NICE draft guidelines or not, there are some valuable lessons to take on board. The peak of EVAR exuberance has probably passed, at least for the current device iteration, and its continued use must be tempered to take account of durability. We need to ensure that training still reflects a future (at least for now) need for open surgery for some patients and accept that such procedures will be technically more difficult. Further centralisation and the proposed LANs may facilitate this. We also need to identify those patients who will not benefit from treatment either because of limited life expectancy or because of comorbidities. Although clearly sensible on a population basis, this is harder to apply to an individual patient and we will need to develop strategies to manage patient expectations and concerns.

Longer term we need to re-evaluate the EVAR concept. The goal is to prevent or effectively treat acute aneurysm rupture with minimal harm to the patient. Certainly, re-lining the aorta with a prosthetic introduced endoluminally remains a very attractive option and is likely to be the way forward, but the current strategy of fixation and radial force without sac management is unlikely to improve outcomes in adverse anatomy. The majority of devices currently available are developments of this original concept and the one deviation away from it (using polymer filled bags in the aneurysm sac) was found wanting. Future devices will need to change and adapt to our knowledge of neck dilatation and changes within 
the sac overtime. The drive should be towards ideally obliteration of the space within the aortic sac rather than with sac size stability.

In conclusion this is not the end for EVAR - there are patient groups that will benefit with today's technology and we have to identify them and continue to monitor them for effectiveness and durability. Crucially, this will enable continued research and development to produce the next iteration of the EVAR concept that will hopefully address some of the current inadequacies. The future may well be endovascular, but it is not yet clear when that will be. In the interim, we need to ensure we have surgeons who can still continue to operate in these challenging patients.

Funding This study was not supported by any funding.

\section{Compliance with Ethical Standards}

Conflict of interest The authors declare that they have no conflict of interest.

Ethical Approval For this type of study informed consent is not required.

\section{References}

1. Brown LC, Powell JT, Thompson SG, Epstein DM, Sculpher MJ, Greenhalgh RM. The UK EndoVascular Aneurysm Repair (EVAR) trials: randomised trials of EVAR versus standard therapy. Health Technol Assess. 2012;16(9):1-218. https://doi.org/10.3310/hta16090.

2. Patel R, Sweeting MJ, Powell JT, et al. Endovascular versus open repair of abdominal aortic aneurysm in 15 -years' follow-up of the UK endovascular aneurysm repair trial 1 (EVAR trial 1): a randomised controlled trial. Lancet. 2016;388(10058):2366-74.

3. Van Schaik TG, Yeung KK, Verhagen HJ, et al. Long-term survival and secondary procedures after open or endovascular repair of abdominal aortic aneurysms. Eur J Vasc Endovasc Surg. 2017;54(5):671.

4. Michel M, Becquemin JP, Clément MC, Marzelle J, Quelen C, Durand-Zaleski I, WINDOW Trial Participants. Editor's choice-thirty day outcomes and costs of fenestrated and branched stent grafts versus open repair for complex aortic aneurysms. Eur J Vasc Endovasc Surg. 2015;50(2):189-96. https://doi.org/10.1016/j.ejvs.2015.04.012.

5. NICE guidelines consultation. Abdominal aortic aneurysm: diagnosis and management. https://www.nice.org.uk/guidance/gid-cgwave0769/ documents/evidence-review-11. Accessed 1 Oct 2019.

Publisher's Note Springer Nature remains neutral with regard to jurisdictional claims in published maps and institutional affiliations. 